\title{
Kernos
}

Revue internationale et pluridisciplinaire de religion grecque antique

12| 1999

Varia

\section{GOODISON, Chr. Morris (éds), Ancient Goddesses. The Myths and the Evidence}

\section{Vinciane Pirenne-Delforge}

\section{OpenEdition \\ Journals}

\section{Édition électronique}

URL : http://journals.openedition.org/kernos/729

DOI : $10.4000 /$ kernos.729

ISSN : 2034-7871

\section{Éditeur}

Centre international d'étude de la religion grecque antique

\section{Édition imprimée}

Date de publication : 1 janvier 1999

Pagination : 301-303

ISSN : 0776-3824

\section{Référence électronique}

Vinciane Pirenne-Delforge, «L. Goodison, Chr. Morris (éds), Ancient Goddesses. The Myths and the Evidence », Kernos [En ligne], 12 | 1999, mis en ligne le 13 avril 2011, consulté le 11 mars 2021. URL : http://journals.openedition.org/kernos/729 ; DOl : https://doi.org/10.4000/kernos.729 
Kernos, 12 (1999), p. 301-341.

\title{
Revue des Livres
}

\section{Comptes rendus et notices bibliog1‘aphiques}

\author{
Lucy Goodison, Christine Morris (éds), Ancient Goddesses. The Myths and \\ the Evidence, British Museum Press, 1998. 1 vol. $18 \times 24,5 \mathrm{~cm}, 224$ p. ISBN : 0- \\ 7141-1761-7.
}

Le but de cet ouvrage à la présentation irréprochable est ambitieux. Il s'agit de rencontrer, sur le terrain scientifique, le "Goddess movement », c'est-à-dire un courant aux composantes très variées qui voit aux origines de la société humaine et de la religion le culte d'une déesse dans une communauté " peace-loving ", égalitaire, matriarcale. Un des objectifs d'une telle perspective est clairement la réappropriation du passé aux fins d'une revendication féministe. Les éditrices du volume souhaitaient dépasser les aspects polémiques du débat en le recentrant autour d'une approche archéologique rigoureuse. Pour ce faire, elles ont invité dix spécialistes de divers domaines de recherche à exposer le plus clairement et le plus objectivement possible le matériel dont ils disposent pour évaluer - et dévaluer le cas échéant - la place des déesses à l'aube des sociétés qu'ils étudient. Cherchant ainsi à rompre le silence académique sur les principales dérives scientifiques de ce courant, les éditrices, avant de passer la parole aux différents «experts ", en retracent l'historiographie, depuis Bachofen, Frazer, Freud, jusqu'aux ouvrages de J. Mellaart et de M. Gimbutas. Conscientes de la difficulté du sujet, elles mettent finement en évidence la nécessité d'une approche souple et fluide, résolument indépendante de tout "fantasme ", qu'il soit féminin ou masculin, et de toute prétention à la création de modèles « universels » qui reflètent davantage des besoins modernes que la grande complexité des faits.

C'est tout à fait dans le sens de cette dénonciation que se place la réflexion de Ruth Tringham et Margaret Conkey (Retbinking figurines. A Critical View from Archaeology of Gimbutas, the 'Goddess' and Popular Culture). Elles stigmatisent la réduction d'idées ou d'objets complexes en quelques caractéristique simples, issues de questions simplificatrices et de prémices imposées par le seul fait de l'autorité de l'archéologue et, ensuite, par la seule vertu de la répétition. Les " idoles " féminines sont un des points nodaux du débat. Les deux A. montrent combien ces artefacts ont échappé aux questions que l'on pose habituellement aux trouvailles archéologiques, comme par l'effet d'une charge émotive particulière attachée à la représentation humaine. Elles présentent alors l'analyse du site préhistorique d'Opovo (ex-Yougoslavie) en replaçant les figurines mises au jour dans toute la richesse de leur contexte. Il ne s'agit plus de poser la question de ce que disent ces figurines sur la femme, mais bien celle de leur sens dans le cadre plus large des besoins humains, des relations qu'elles présupposent dans la société au sens large. Le tableau qui en résulte est bien plus nuancé, même s'il exorcise moins que d'autres l'horreur du vide. 
La fouille menée dans les années ' 60 sur le site néolithique de Çatalhöyük par J. Mellaart a beaucoup contribué à la conception d'une «Grande-déesse-mère » préhistorique et à celle d'une continuité allant de l'Anatolie aux Grecs, les dépositaires de la culture occidentale dont nous sommes les héritiers. Lynn Meskell (Twin Peaks. The Archaeologies of Catalböyilk) propose une analyse très intéressante de la logique circulaire qui a prévalu dans l'analyse du site et de l'iconographie mise au jour. Çatalhöyük est devenu le lieu de toutes les projections anachroniques ( « an archaeology of desire »). Il est dommage que l'A. n'aborde pas la bibliographie francophone : elle y aurait trouvé maintes illustrations supplémentaires de son propos.

C'est ensuite au Proche-Orient qu'est réaffirmée la nature profondément polythéiste des systèmes religieux qui s'y sont développés entre 3000 et 1000 avant notre ère (Joan Goodnick Westenholz, Goddesses of the Ancient Near East 30001000 b.C.). L'A. réagit contre le "filtre monothéiste » qui a fait apparaître une «Grande déesse » en effaçant l'individualité des nombreuses divinités féminines par une sorte de réduction des unes aux autres. De plus, si certaines d'entre elles assument effectivement les rôles féminins traditionnels, d'autres les ignorent. Karel van den Toorn (Goddesses in Early Israelite Religion) jette quant à lui un éclairage très intéressant sur l'existence d'une (ou de plusieurs) « reine(s) des Cieux » parèdre du Yahweh des Hébreux, à l'instar d'autres couples de ce type dans les systèmes religieux de la région. Il conclut à l'existence, dans le cadre d'une dévotion plus populaire, d'une personnalité divine médiatrice, un peu comme «la face humaine » de Dieu. Pour l'Égypte, Fekri A. Hassan (The Earliest Goddesses of Egypt. Divine Mothers and Cosmic Bodies), tout en rendant bien compte de la multiplicité des figures divines, ne me semble pas avoir échappé totalement aux travers dénoncés par les éditrices : trop de «psychologie », de surinterprétations "préhistoriques » et de schémas convenus rendent le lecteur mal à l'aise.

Pour l'ouest du bassin méditerranéen, trois articles concernent respectivement l'île de Malte, un des terrains de prédilection de l'approche «Déesse-mère » (Caroline Malone, God or Goddesses. The Temple Art of Ancient Malta), la France (Elizabeth Shee Twohig, A 'Mother Goddess' in North-West Europe c. 4200-2500 B.C. ?), et l'âge du Fer en Gaule et en Grande-Bretagne (Miranda J. Green, Some Gallo-British Goddesses. Iconography and Meaning). Chacune de ces études évalue avec précision l'apport de l'archéologie à la connaissance des sociétés dont elle traite, et plus précisément à la pertinence du concept de «Grande-déessemère $»$.

Restent deux des articles, qui intéresseront tout particulièrement les lecteurs de Kernos. Lucy Goodison et Christine Morris ont placé dans le cadre de la civilisation minoenne la problématique du volume dont elles sont les éditrices (Beyond the 'Great Mother'. The sacred world of the Minoans). Arthur Evans a hérité de J.G. Frazer l'image de la «Déesse », là où M.P. Nilsson parlait déjà de panthéon. La prédominance de l'image féminine est impressionnante, mais il ne faut pas négliger la question difficile de l'identité de ces figures, dont le caractère divin n'est ni évident ni, sans doute, systématique. De plus, les intérêts rituels diversifiés révélés par les trouvailles dans les tombes invitent à ne pas souscrire trop rapidement à une sorte de "monothéisme anthropomorphique féminin » qui fait fi des dieux masculins et des manifestations animales, ou simplement naturalistes, du divin. La période post-palatiale (1450-1050) connaît de profonds changements dans l'expression religieuse : nouvelles localisations, grandes figurines féminines en terre cuite au centre des «bench-sanctuaries », utilisation d'an - 
ciennes images, comme les animaux, les doubles cornes, les doubles haches, non plus comme symboles indépendants mais comme attributs.

Pour la Grèce, c'est Mary Voyatzis qui pose la question d'un éventuel héritage assumé par les déesses grecques des périodes archaïque et classique (From Athena to Zeus. An A-Z Guide to the Origins of Greek Goddesses). Parfaitement consciente du caractère périlleux d'une réflexion globale et théorique sur le sujet, elle propose l'analyse précise du site d'un sanctuaire particulier, celui d'Athéna Aléa à Tégée où elle a fouillé en collaboration avec les Norvégiens. La présentation du matériel mis au jour et son interprétation mesurée permettent de dessiner l'image d'une déesse dont les intérêts diversifiés ont évolué au fil du temps, en s'adaptant aux besoins des fidèles. Aléa pourrait être l'ancien nom de la déesse en question. Son association à Athéna ne semble pas antérieure à la fin du $\mathrm{VII}^{\mathrm{e}}$ ou au début du $\mathrm{VI}^{\mathrm{e}}$ siècle. Elle propose ensuite un bref panorama des autres divinités d'Arcadie, essentiellement fondé sur l'ouvrage de M. Jost, Sanctuaires et cultes d'Arcadie (Paris, 1985), puis elle élargit le cadre à d'autres régions de Grèce, comme pour justifier le titre de l'article, « un guide de A à $Z$ ». Le propos se fait alors plus englobant : la chute des palais mycéniens a entraîné en certains lieux l'arrêt manifeste des activités cultuelles, qui se sont pourtant poursuivies dans d'autres. L'isolement des communautés entre le $\mathrm{xI}^{\mathrm{e}}$ et le $\mathrm{Ix}^{\mathrm{e}}$ siècle a entraîné des variations dans le développement religieux à l'échelon local, encore accrues par l'arrivée de nouveaux venus, porteurs d'autres conceptions et pratiques religieuses. La conclusion de l'étude recueille les fruits de cette approche mesurée et prudente : au début de l'époque archaïque, beaucoup de divinités sont honorées dans les grands sanctuaires, mais ceux des déesses sont incontestablement plus nombreux et plus importants. Apollon est le seul dieu masculin à jouer un rôle essentiel à l'époque. Il est concevable que la prédominance des déesses et les traits communs qu'elles partagent soient un héritage de la période mycénienne.

Cet ouvrage est clair, bien documenté, et il offre une vision équilibrée d'une problématique qui a longtemps empoisonné l'histoire des religions en général, et de la religion grecque en particulier. En témoigne encore un ouvrage récent dédié à la mémoire de Maria Gimbutas et au titre significatif : Die Madonna und ibre griecbiscben Töchter ${ }^{1}$.

Vinciane Pirenne-Delforge (FNRS - Université de Liège)

Nicolas Chr. Stampolidis, Anti Poina. "Reprisals". Contribution to the study of customs of the geometric-archaic period, Rethymno, University of Crete, 1996. 1 vol. $23 \times 29 \mathrm{~cm}, 253$ p., 224 fig. 2 pl. (Eleutherna. Sector III, 3).

Vu que la fouille de la nécropole d'Eleutherna, en Crète occidentale, est toujours en cours, on comprend l'embarras des chercheurs désireux d'interpréter les vestiges anciens découverts là-bas. En effet, les suggestions proposées risquent d'être bouleversées par des découvertes à venir.

Pourtant, dans cette étude, l'A. entreprend la tâche difficile de discuter un charnier de fouille, dont la mise au jour n'a pas été complétée. Dans l'introduction de son livre [p. 15], il souligne que son but est de mettre en évidence une découverte particulière, qui risquerait de passer inaperçue jusqu'à la publication

1 Harald HaArmann, Die Madonna und ibre griecbiscben Töchter. Rekontruktion einer kulturbistoriscben Genealogie, Hildesheim, Zürich, New York, Georg Olms Verlag, 1996 (compte rendu à paraître dans L'Antiquité Classique, 68 [1999]). 